\title{
'Guardate qui, mucchio di casinari da cantina': Bob Dylan al lavoro sottoterra
}

\author{
Alessandro Bratus \\ alessandro.bratus@gmail.com \\ Università di Pavia (Cremona)
}

\begin{abstract}
I Basement Tapes di Bob Dylan si situano in un periodo cruciale della carriera del cantante, durante il suo passaggio da una produzione basata sui modelli del folkrevival al suono elettrico delle performance e dei dischi del 1965. In questo articolo una breve panoramica analitica su quattro canzoni fornirà le indicazioni fondamentali per contestualizzare questo repertorio all'interno della carriera di Dylan e della storia della canzone popular americana, dal punto di vista musicale, stilistico e ideologico.
\end{abstract}

Key words: The Band; Basement Tapes; popular music americana; folk-revival; analisi della canzone; processo creativo; composizione nella popular music; metodo analitico.

\section{Introduzione}

I Basement Tapes di Bob Dylan si situano in un periodo cruciale della carriera del cantante, durante il suo passaggio da una produzione basata sui modelli del folk-revival a nuove modalità di scrittura. Le loro radici possono essere ricondotte alle sonorità presentate nelle registrazioni del 1965, anno durante quale - a luglio - Dylan si presentò persino al Newport Folk Festival accompagnato da una band elettrica. L'ormai ex-"portavoce di una generazione", come lo soprannominarono i fans e i critici per il suo impegno a favore delle cause politiche e sociali, sembrava essere sul punto di tradire definitivamente le sue stesse radici culturali. Ciò fu confermato, nei mesi immediatamente successivi, dall'inizio di un nuovo tour i cui show erano divisi in due parti: la prima acustica, con solo il cantante e la sua chitarra sul palco, la seconda insieme al complesso The Hawks, normalmente accolti dal pubblico con rumorose proteste. Come già accaduto a Newport, anche in questi casi il motivo del contendere era 
legato all'interpretazione ideologica della sua musica. I critici e gli appassionati che lo avevano accolto come una tra le voci più importanti del movimento folkrevival percepivano il suo repentino passaggio ai suoni elettrificati come un rifiuto di confermare quelle scelte di campo politiche e sociali fondamentali nella prima fase della vicenda artistica del cantante.

Questo vero e proprio tour de force fu interrotto bruscamente da un incidente motociclistico occorso nel giugno 1966, che costrinse Dylan a prolungare la sua permanenza nella cittadina di Woodstock, dove si stava riposando in vista della tournée americana che sarebbe iniziata entro poche settimane. Sebbene né il cantante né il suo management abbiano mai fatto chiarezza su tutti i particolari di questo evento, sicuramente questo fu la causa scatenante di un ritiro dai palcoscenici durato ben otto anni, fino al 1974. La Iontananza fisica dal pubblico non significò un totale silenzio creativo, anzi si possono far risalire a questo periodo pubblicazione discografiche del calibro di John Wesley Harding o Nashville Skyline, oltre alla composizione - insieme alla sua band - di un gran numero di canzoni conosciute nel complesso con il nome di Basement Tapes. Si tratta di una raccolta di circa 130 pezzi, più o meno equamente divisi tra nuove creazioni e cover provenienti dai più diversi generi della canzone americana. Il loro nome deriva dalle inusuali circostanze della loro realizzazione, interamente consumata tra le pareti delle case dei musicisti a Woodstock, riadattando a provvisorio studio d'incisione stanze dismesse e cantine.

\section{In cerca di un metodo: fondamenti teorici e strumenti dell'analisi}

Oltre a trovarsi in un momento centrale della biografia di Dylan e di The Band, i Basement Tapes devono il loro interesse come oggetto di studio scientifico soprattutto alla loro qualità ibrida, oggetto che si può avvicinare tanto con gli strumenti della musicologia, quanto con quelli dell'etnomusicologia. La loro analisi, quindi, dovrà necessariamente essere preceduta da una discussione del loro status, in grado di armonizzare gli scopi di uno studio musicologico con le caratteristiche del materiale preso in esame. Per un inquadramento generale di tale problema si è fatto riferimento al "triangolo assiomatico" proposto da Philip Tagg (1994, p. 48), dal quale risulta chiaramente l'ambiguità dei Basement Tapes, impossibili da far ricadere con sicurezza in una delle due categorie di musica folk o popular. Con il primo termine Tagg si riferisce a quelle pratiche musicali trasmesse oralmente e appartenenti a una comunità locale ben definita; mentre con il secondo alla musica prodotta in seno alle società industriali e basata sulla trasmissione mass-mediatica, nella maggioranza dei casi tramite un supporto fonografico.

Nel complesso l'unica caratteristica comune tra musica tradizionale e popular sembra essere la mancanza di un sistema teorico ed estetico determinato a priori, una circostanza legata al comune riferimento a diverse tipologie di trasmissione orale. Per il resto le discriminanti citate da Tagg si 
trovano ad essere irrimediabilmente confuse nei Basement Tapes, tanto che nessuna di esse, di per sé, può definire in modo soddisfacente la totalità di queste registrazioni. A partire dalle loro modalità di produzione e trasmissione: se da una parte le canzoni sono il risultato di una collaborazione tra professionisti, dall'altra parte si possono anche considerare come frutto di un approccio quasi amatoriale, come dimostra tra le altre cose l'atteggiamento rilassato dei musicisti durante le prove - favorito anche dall'assenza di un produttore o di un manager incaricato di seguire i lavori. Da un punto di vista storico, questo è un fatto non totalmente nuovo nella produzione del rock anglo-americano, che proprio in questi anni vede un'analoga tendenza da parte degli artisti ad avvicinarsi in modo più consapevole alle potenzialità artistiche dei mezzi di produzione e postproduzione, con esempi che vanno da Sgt. Pepper's Lonely Hearts Club Band dei Beatles (1967) a Electric Ladyland di Jimi Hendrix (1968), tra gli altri. ${ }^{1}$

Tabella 1: Affinità e divergenze tra folk e popular music, adattata da Tagg (1994, p. 48).

\begin{tabular}{|l|l|l|}
\hline Caratteristiche & Folk & Popular \\
\hline - Prodotta e trasmessa & $\begin{array}{l}\text { principalmente da } \\
\text { dilettanti }\end{array}$ & $\begin{array}{l}\text { principalmente da } \\
\text { professionisti }\end{array}$ \\
\hline - Distribuzione di massa & rara & consueta \\
\hline $\begin{array}{l}\text { - Principali modalità di conservazione e } \\
\text { trasmissione }\end{array}$ & trasmissione orale & suono registrato \\
\hline - Tipo di società nella quale si sviluppa & nomade o contadina & industriale \\
\hline $\begin{array}{l}\text { - Presenza di una teoria o di un'estetica } \\
\text { scritta }\end{array}$ & rara & rara \\
\hline - Compositore/autore & anonimo & non-anonimo \\
\hline
\end{tabular}

Nei Basement Tapes, però, è importante sottolineare come questo non sia un modo per esplorare le possibilità espressive dei nuovi strumenti elettronici, o per realizzare un artefatto sonoro ideale, riproducibile solamente con l'incisione fonografica. Al contrario di quanto si può osservare negli esempi contemporanei, questa scelta per Dylan era semplicemente dettata dalla volontà di imporre un ritmo differente alla sua creatività, allontanandosi dagli obblighi e dalle sessioni rigidamente fissate degli studi ufficiali della Columbia e recuperando un concetto quasi artigianale di elaborazione delle proprie canzoni. Questo mutato approccio al lavoro in studio fa il paio con le modalità compositive di questo repertorio, che prevedono certamente la messa in campo da parte dei loro autori delle proprie competenze professionali sotto il profilo della strumentazione e dell'arrangiamento, ma sempre sulla base di un'armatura di fondo composta dal cantante, trasmessa personalmente da questi ai suoi compagni. La stessa ambivalenza si ripropone considerando la distribuzione; vista la loro originaria destinazione come numeri da proporre per una reinterpretazione da parte di altri artisti e gruppi, i Basement Tapes non possono non essere inseriti in un sistema di produzione culturale massificato, mentre d'altra parte la loro circolazione ristretta a una piccola comunità d'ascolto li riconduce verso un ambito lontano dalla categoria del popular in senso stretto. Dal punto di vista del supporto su cui 
vengono conservate, se la registrazione è per questo repertorio essenziale, come per la gran parte della popular music contemporanea, si è già sottolineato come questa sia preceduta dal passaggio, per via orale, delle principali linee stilistiche e formali del pezzo dal cantante ai The Hawks. Rimane infine da considerare il discorso dell'autorialità di queste canzone, un altro campo in cui distinguere i due ambiti culturali non è così semplice. Nonostante sotto il profilo strettamente legale i numeri dei Basement Tapes siano accreditati in massima parte al solo Dylan (in rari casi in collaborazione con altri membri di The Band), è grazie allo sviluppo collettivo, per forza di cose anonimo, avvenuto nelle cantine di Woodstock che l'idea iniziale si è potuta trasformare nel risultato sonoro testimoniato dall'incisione. Ciò è testimoniato in maniera particolarmente chiara dai pezzi registrati in più di una versione (come ad esempio Tears Of Rage, di cui ci si occuperà brevemente nell'ultimo paragrafo), che fotografano su nastro fasi diverse della loro messa a punto.

Una volta chiarite tali considerazioni di fondo, il metodo analitico è stato elaborato tenendo presente lo specifico interesse musicologico per questi oggetti in quanto artefatti sonori, testimonianze di una performance avvenuta in un preciso contesto. In questo modo diventa possibile avvicinarsi ad esse utilizzando gli strumenti della musicologia "classica", ovviamente adattandoli alle specificità della popular music e cercando di mantenere al minimo le interferenze di una terminologia di un campo di studi sviluppatosi originariamente per l'indagine sulla musica d'arte dell'Occidente. Questo tipo di atteggiamento è particolarmente importante in un campo, come quello dei popular music studies:

[...] dominated by cultural critics and sociologists, many of whom do not possess the specialized skills necessary to deal with the musical 'texts' in the ways that musicologists do. These scholars tend to be concerned with the effect of musics on listeners, and on the ways in which musics can have various kinds of significance within cultures and subcultures; and they treat the music, in the words of John Sheperd, as a kind of 'inscrutable black box' (Covach, 2001, pp. 454-455).

Ribaltando un paradigma molto diffuso all'interno della disciplina, Covach rivendica il ruolo cruciale della musicologia per un'indagine che consideri la popular music "as inherently musical, and only secondarily social" (ibid., p. 466). Una simile posizione è stata fondamentale nell'indirizzare la costruzione teorica di un possibile metodo analitico per i Basement Tapes, nel quale: "The challenge then becomes the investigation of popular music along traditional musicological lines while maintaining a careful sensitivity to how popular music may differ from art-music in its specifically musical dimensions" (ibid., p. 466). Così facendo, l'intento non è quello di "legittimare" la popular music in un settore disciplinare già profondamente connotato in termini di giudizi di valore, bensì quello di includere il mio oggetto di interesse all'interno di un sistema di conoscenze che è già possiede tutti gli strumenti concettuali per spiegare il funzionamento e le strutture delle canzoni di Bob Dylan \& The Hawks, se non dell'intero repertorio rock angloamericano. La questione, allora, non tanto è quella di ridefinire la musicologia per 
intero, quanto comprendere in che modo i concetti sviluppati per lo studio della musica colta possano essere applicati a diverse pratiche culturali. La mia speranza nel proporre un simile percorso non è, in sintesi, tanto quella di "gettare un ponte" tra popular music studies e musicologia, come propone Richard Middleton (2002), bensì contribuire ad abbattere i confini tra le due, formatisi in massima parte per effetto dei persistenti pregiudizi culturali di una parte della ricerca accademica nei confronti della cultura popolare contemporanea. Come scrive Stefano La Via nel suo volume dedicato alle relazioni tra musica e poesia, interamente dedicato a oggetti riconducibili a entrambi i campi culturali:

Troppo spesso gli studiosi impegnati nei due rispettivi versanti tendono, ancora oggi, a chiudersi nei loro angusti e specialistici abitacoli disciplinari, contribuendo solo ad innalzare e rafforzare quelle stesse muraglie cinesi che da sempre tutti i grandi letterati, compositori e poeti cantanti si sono sforzati di abbattere, riuscendo non di rado ad aprire autentici quanto fertili e rivitalizzanti varchi di mutua comunicazione (La Via, 2006 [CD-Rom], p. 14).

Data la complessità della canzone come oggetto, inteso nella sua concretezza di incisione su un supporto riproducibile, si è reso necessario considerarla a livello analitico come oggetto multiplanare, nel quale musica e parole lavorano insieme alle caratteristiche stilistiche e sonore per dare origine a diversi livelli di un significato unitario. Ognuno di questi parametri, di per sé, è dotato di una propria logica interna, ma è anche parte di un sistema più ampio, all'interno del quale è possibile rintracciare un criterio unificante che possa comprendere le connessioni, le omologie, i contrasti tra i singoli elementi. Se interpretata in questo modo la canzone diventa, allora, un sistema dinamico, dotato di precise proprietà strutturali, storiche, tecnologiche, tutte convergenti verso un medesimo scopo espressivo. Un tale metodo, basato su una prima frammentazione e su una successiva ricomposizione analitica della totalità del pezzo, è stato ispirato dall'approccio proposto da Rob Bowman nel suo studio sul sound della Stax Records (2002). Dovendosi confrontare con un repertorio prodotto nello stesso luogo, da un numero limitato di persone, con la stessa strumentazione tecnica, lo studioso americano si concentra su nove caratteristiche stilistiche, dalla qualità dell'arrangiamento agli effetti di post-produzione, nel tentativo di trovare cosa definisca, dal punto di vista delle caratteristiche musicali, il suono di quell'etichetta tra il 1960 e il 1974. Dopo aver rielaborato il suo metodo secondo gli scopi della mia ricerca e le specificità dei Basement Tapes, ne è risultato un percorso d'indagine analogamente strutturato per aree di interesse, più ampie e generiche. Questo per superare la principale differenza tra uno studio, come quello di Bowman, focalizzato sul sound e sullo stile, e un'analisi il cui scopo è l'interpretazione complessiva di una canzone, dal punto di vista delle sue strutture interne e della sua posizione nel contesto della tradizione musicale americana. Ne è conseguita un'impostazione analitica su quattro parametri: 
1. costruzione micro- e macro-formale del testo verbale (strofe, ritornelli, rime, metrica, etc.);

2. costruzione musicale, in particolare concentrandosi sull'armonia, sulle tipologie scalari, sull'arrangiamento, sulla struttura della melodia rispetto alle principali cesure del testo verbale;

3. influenze stilistiche, dall'esterno e dall'interno della produzione del cantante;

4. caratteristiche sonore, con un'attenzione specifica per la disposizione spaziale delle fonti sonore e della "scatola sonora" delle registrazioni. ${ }^{2}$

La principale differenza tra i due metodi è l'inserimento, in quello elaborato per i Basement Tapes, della parte focalizzata sul testo verbale, un aspetto fondamentale nella costruzione delle strutture e nei significati delle canzoni di Bob Dylan, oltre a costituire un aspetto della creatività di questo artista tra i più influenti sulle generazioni successive di songwriters e musicisti. Inoltre, come spesso capita nelle sue canzoni, in queste registrazioni si nota una relazione particolarmente stretta tra suono e parola, che compartecipano nella formazione di strutture autenticamente poetico-musicali, importanti non solo sotto il profilo formale, ma anche per le loro implicazioni a livello ideologico ed estetico nel contesto delle composizioni nate nelle cantine di Woodstock.

\section{Quattro esempi analitici per una panoramica sui Basement Tapes}

Tenendo presenti i presupposti teorici appena esposti, e dopo uno screening a largo raggio sull'intero corpus dei Basement Tapes, si è scelto di concentrare l'attenzione su quattro canzoni in particolare, ciascuna indicativa di un aspetto rilevante per la definizione complessiva di queste registrazioni. Gli oggetti di analisi sono stati selezionati per il loro valore paradigmatico, in quanto rappresentativi di concetti centrali nella comprensione della produzione di Dylan \& The Hawks nel 1967. Per questa ragione la presentazione delle principali conclusioni raggiunte dalla mia ricerca sui Basement Tapes può essere condotta ripercorrendo le più interessanti caratteristiche formali, stilistiche e sonore di questi esempi, interpretati alla stregua di chiavi analitiche, capaci di suggerire le linee guida per un'interpretazione globale del repertorio esaminato.

\section{1. 'Apple Suckling Tree' e l'eredità del folk}

II primo caso è quello di 'Apple Suckling Tree', una canzone originale basata sul riadattamento della nursery rhyme inglese 'Froggie Went A-Courtin". 3 Questo conferma la persistente importanza del materiale tradizionale come 
ispirazione per le canzoni di Bob Dylan, anche in momento della sia vicenda artistica e biografica in cui sembrava aver abbandonato l'ambiente e le coordinate culturali del folk-revival. Se questo è vero sotto il profilo personale e ideologico, sotto quello musicale i Basement Tapes dimostrano una continuità ininterrotta con la sua produzione precedente. Come già accadeva nei suoi primi album, la canzone folk è sottoposta a un processo di radicale revisione e appropriazione creativa, un processo essenziale in tutta la carriera del cantante per prendere possesso di questo materiale e, contemporaneamente, metterlo nelle condizioni di parlare il linguaggio della contemporaneità.

Anche a un primo esame le due canzoni dimostrano il loro legame, come indicano chiaramente la somiglianza nel profilo melodico complessivo, il testo dedicato al racconto di situazioni fantastiche, l'unione tra l'idioma musicale tradizionale proveniente dall'Europa e quello del blues. Soprattutto quest'ultimo punto è importante da sottolineare, dato che in tutti i Basement Tapes il blues sembra essere una sorta di lingua franca, un sistema espressivo e formale che permette a Dylan di mescolare liberamente i diversi generi e stili della canzone americana, nella ricerca di un proprio idioletto. In 'Apple Suckling Tree' l'influenza del genere afroamericano è resa palese da elementi quali l'adozione della forma $A A B$ delle strofe, della sequenza accordale tipica, dell'intelaiatura modale della melodia (con l'impiego di quella che Gerhard Kubik chiamerebbe una "terza flessibile" fa-fa\#), ${ }^{4}$ della costruzione antifonale delle parti di voce e organo. La struttura testuale di tipo $A A B$ è una caratteristica tipica delle stanze blues, che come nel caso della canzone di Dylan si accompagna a un pattern armonico ripetuto e uguale per tutte le strofe. Come scrive a questo proposito Paul Oliver definendo tale forma nella più recente edizione online del The New Grove Dictionary of Music and Musicians:

Apparently this form crystallized in the first decade of the 20th century as a three-line stanza in which the second line repeated the first, thus enabling the blues singer to improvise a third, rhyming line while singing the second:

I'm troubled in mind, baby, feelin' blue and sad /

I'm troubled in mind, baby, feelin' blue and sad /

The blues ain't nothin' but a good man feelin' bad.

(Oxford Music Online, 2009)

L'esempio proposto da Oliver può essere facilmente confrontato con la strofa di 'Apple Suckling Tree', suddivisa come nella Tabella 2:

Tabella 2: La strofa di 'Apple Suckling Tree' ricondotta allo schema AAB.

\begin{tabular}{|l|l|}
\hline A & Old man sailin' in a dinghy boat down there \\
\hline A & Old man down is sailin' a hook on there \\
\hline B & $\begin{array}{l}\text { Gonna pull man down on a suckling hook } \\
\text { And under basin of a peeling brook } \\
\text { Oh yeah! }\end{array}$ \\
\hline
\end{tabular}

I@J vol.1, no.1 (2010) http://www.iaspmjournal.net 
I primi due versi ripropongono, leggermente variato, uno stesso elemento, mentre i tre versi finali concludono la sezione; tale soluzione è comune sia alla strofa, sia al ritornello della canzone di Dylan, musicato sulla base della stessa sequenza melodico-armonica e distinto dalla prima sezione solo dalla ripetizione letterale del testo verbale, un altro elemento che contribuisce ad avvicinare al blues la costruzione di 'Apple Suckling Tree'. A questo proposito è anche interessante sottolineare come vi sia una significativa corrispondenza tra lo svolgimento temporale del pattern accordale e quello delle singole sezioni formali. La parte B della strofa, infatti, coincide con la cadenza finale dello schema blues (IV-I-V-I), un dato enfatizzato nel ritornello anche dal break strumentale che improvvisamente svuota la struttura sonora, lasciando per un paio di battute solamente la voce e l'accompagnamento di batteria. E' questo un esempio delle omologie strutturali, con un chiaro valore retorico ed espressivo, che si possono ritrovare nelle composizione dylaniane, segno di quella logica poetico-musicale riscontrabile ad ampio raggio nella sua scrittura. Inoltre, un esame diretto delle sequenze armoniche delle due canzoni permette di rendersi conto della stretta somiglianza tra la sequenza armonica della canzone di Dylan e quello tipico della tradizione afroamericana.

Tabella 3: Comparazione tra le sequenze accordali di 'Apple Suckling Tree' e del blues in 12 battute.

\begin{tabular}{|c|c|c|c|c|}
\hline $\bar{D}$ & $\mathrm{D}$ & $\mathrm{D}$ & $A$ & $\rightarrow(\mathrm{I}-\mathrm{V})$ \\
\hline D & $G$ & $D-A$ & D & $\rightarrow(\mathrm{I}-\mathrm{IV}-\mathrm{I}-\mathrm{V}-\mathrm{I})$ \\
\hline
\end{tabular}

\begin{tabular}{|l|l|l|l|}
\hline I & I & I & I \\
\hline IV & IV & I & I \\
\hline V & V & I & I \\
\hline
\end{tabular}

Nella Tabella 3 la concatenazione accordale di 'Apple Suckling Tree' è messa a confronto con il modello blues in 12 battute definito da Peter Van Der Merwe "standard blues pattern", dal quale discendono per diminuzione o aumentazione le forme in 8 (ad esempio il "Gregory Walker pattern") o 16 battute, interpretate come varianti dello stesso schema originario (1994, pp. 198-204). Proprio per tale ragione è interessante osservare come Dylan si approcci a una struttura "universale" nella musica americana e ne faccia uno strumento al servizio dei suoi scopi espressivi, modificandolo leggermente, ma in maniera decisiva. Dopo aver mantenuto la struttura fondamentale delle prime quattro battute, il cantante sintetizza nello spazio delle quattro battute seguenti l'intera cadenza IV-I-V-I, dimezzando la durata di ogni cambio di accordo. Così facendo Dylan si fa portatore di un processo di elaborazione che seleziona e procede alla creazione 
di nuovi oggetti attraverso l'ibridazione tra caratteristiche riprese dalle fonti più diverse, i cui elementi-base sono riconfigurati all'interno di un oggetto provvisto di peculiarità originali:

One of the great forces in American music, both white and black, but especially black, is what one could call creative fragmentation. A tune is broken down into simpler and simpler elements, while, in compensation, these elements may develop a new complexity of their own (Van der Merwe, 1994, p. 203).

Infine, un esempio musicale tratto da questa canzone servirà per illuminare l'ultima particolarità organizzativa che 'Apple Suckling Tree' condivide con il blues, cioè l'uso di una tecnica call and response di cui sono protagonisti la voce e la linea dell'organo. Tutto questo insieme di elementi musicali, oltre a segnalare la vicinanza con il genere afroamericano, ne ribadiscono la centralità come linguaggio in grado di far convivere in una stessa canzone, e nei Basement Tapes come repertorio nel suo complesso, tratti provenienti da contesti culturali anche molto diversi in un idioletto personale e originale.

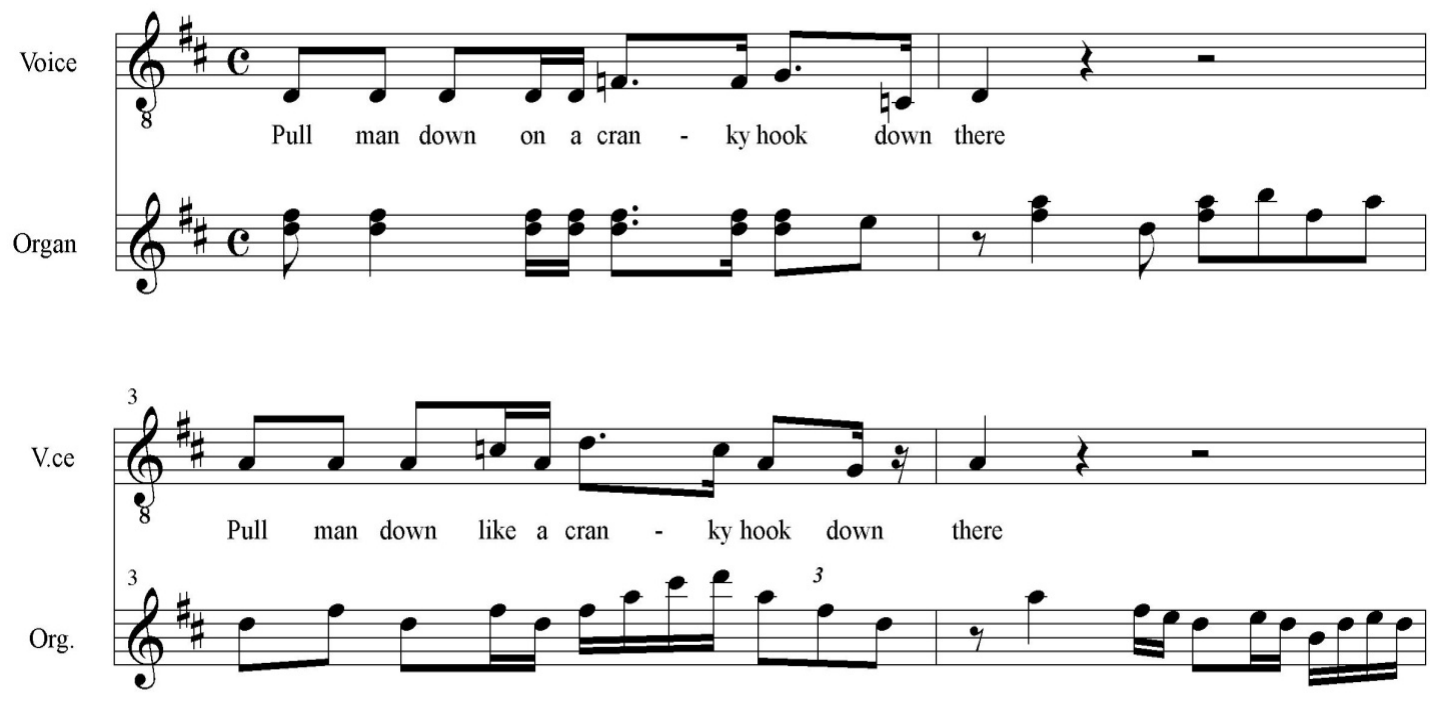

Figura 1: 'Apple Suckling Tree' \#2, bb. 12-15 (voce e organo).

\section{2. 'Clothesline Saga': intensionalità nella composizione popular}

Un esempio simile di songwriting ispirato a materiale musicale e verbale preesistente è rappresentato dalla seconda canzone presa in esame, ma questa volta la relazione è con una canzone contemporanea, una hit da classifica 
dell'estate 1967: 'Ode to Billie Joe' di Bobbie Gentry. Come nel caso precedente Dylan riesce anche qui a comporre una canzone "originale", risultante non solo dalla somma tra le parti riprese dal suo modello, ma una nuova entità nella quale tali componenti sono inscritte in un sistema coerente. Da questo punto di vista entrambe le canzoni possono essere interpretate come prodotti di meccanismi di "auto-riflessione", comuni tanto nella produzione folklorica, quanto in quella popular. Tali legami intertestuali sono stati interpretati da Richard Middleton come dispositivi basilari nella costruzione del significato di una canzone, a livello formale, stilistico e ideologico (1994, p. 302). Allargando le considerazioni dello studioso inglese all'intero campo della popular music, questa può essere interpretata nel suo insieme come frutto di fenomeni intertestuali a largo raggio, in cui è possibile ritrovare una serie di possibili modalità di collegamento, dalla citazione letterale alla velata allusione. Inoltre, ritrovare il riferimento a modelli compositivi così diversi ha permesso di rendersi conto dell'ampiezza degli orizzonti musicali dei partecipanti alle registrazioni di Woodstock, che spaziano dal riadattamento di una nursery rhyme tradizionale alla parodia di un successo commerciale contemporaneo. II loro atteggiamento compositivo non sembra essere molto diverso in questi due casi così distanti a livello cronologico e culturale: gli elementi costitutivi tratti dalle diverse fonti sono trattati, selezionati, rielaborati secondo logiche analoghe, senza riguardo per la loro provenienza. Ciò è ancora confermato, per un altro verso, anche dalla varietà delle cover incise durante le registrazioni del 1967 di Dylan \& The Band, che appartengono a tutti i generi più rappresentativi della canzone americana. Presentando la panoramica complessiva contenuta nella Tabella 4 , si vuole precisare che la classificazione non vuole offrire una rigida categorizzazione delle canzoni (su cui si potrebbe discutere), quanto indicare il loro riferimento a un determinato stile musicale e ai suoi interpreti più rappresentativi.

Le osservazioni precedenti mi hanno portato a concepire il lavoro compositivo portato avanti in queste repertorio come principalmente "intensionale", secondo la definizione presentata da Andrew Chester nel saggio For a Rock Aesthetic (1970). Commentando tale articolo, Allan Moore ha scritto:

Chester distinguished between 'classical' music (intensional composition), which consisted of small, indivisible musemes which were put together in additive fashion to form musical pieces, and 'blues-derived' music wherein large-scale structures underwent internal development through quasiimprovisatory practices (extensional composition). [...] Intensional construction pre-supposes an existent structure which will necessarily have an historical dimension, a dimension absent from the 'extensional' musemes (Moore, 1997). 
Tabella 4: Le cover nei The Basement Tapes.

\begin{tabular}{|c|c|c|c|c|}
\hline Genere & Titolo & Anno & Parole-musica & $\begin{array}{l}\text { Primo, o più famoso } \\
\text { interprete }\end{array}$ \\
\hline \multirow{5}{*}{ Rock'n'roll } & $\begin{array}{l}\text { I Forgot To Remember To } \\
\text { Forget }\end{array}$ & 1955 & $\begin{array}{l}\text { Stan Kesler - Charlie } \\
\text { Feathers }\end{array}$ & Elvis Presley \\
\hline & Confidential & 1956 & Dorinda Morgan & Sonny Knight \\
\hline & Silhouettes & 1957 & $\begin{array}{l}\text { Frank C. Slay Jr. - Bob } \\
\text { Crewe }\end{array}$ & \\
\hline & All American Boy & 1959 & $\begin{array}{l}\text { Bill Parson - Orville } \\
\text { Lunsford }\end{array}$ & \\
\hline & Baby Ain't That Fine & 1966 & Dallas Frazier & Gene Pitney \\
\hline \multirow{10}{*}{ Country } & $\begin{array}{l}\text { Be Careful Of The Stones That } \\
\text { You Throw }\end{array}$ & 1949 & Bonnie Dodd & Little Jimmie Dickens \\
\hline & You Win Again & 1952 & Hank Williams & John Lee Hooker \\
\hline & $\begin{array}{l}\text { (Now And Then There's) A } \\
\text { Fool Such as I }\end{array}$ & 1953 & Bill Trader & Hank Snow \\
\hline & I Don't Hurt Anymore & 1954 & $\begin{array}{l}\text { Don Robertson - Jack } \\
\text { Rollins }\end{array}$ & Hank Snow \\
\hline & Folsom Prison Blues & 1956 & Johnny Cash & \\
\hline & Belshazar & 1957 & Johnny Cash & \\
\hline & Big River & 1958 & Johnny Cash & \\
\hline & Cool Water & 1959 & Bob Nolan & Marty Robbins \\
\hline & Waltzing With Sin $1 \& 2$ & 1963 & Hayes - Burns & Cowboy Copas \\
\hline & Still In Town & & $\begin{array}{l}\text { Hank Cochran - Harlan } \\
\text { Howard }\end{array}$ & \\
\hline \multirow{4}{*}{ Blues } & $\begin{array}{l}\text { You Gotta Kickin' My Dog } \\
\text { Around }\end{array}$ & 1916 & $\begin{array}{l}\text { Webb M. Oungst - Cy } \\
\text { Perkins }\end{array}$ & $\begin{array}{l}\text { Byron J. Harlan and the } \\
\text { American Quartet }\end{array}$ \\
\hline & $\begin{array}{l}\text { See That My Grave Is Kept } \\
\text { Clean }\end{array}$ & 1928 & $\begin{array}{l}\text { trad./ "Blind Lemon" } \\
\text { Jefferson }\end{array}$ & \\
\hline & I'm In The Mood & 1951 & John Lee Hooker & \\
\hline & Flight Of The Bumble Bee & & Trad. & \\
\hline Soul & People Get Ready & 1965 & Curtis Mayfield & The Impressions \\
\hline \multirow{3}{*}{ Folk-revival } & Joshua Gone Barbados & 1963 & Eric Von Schmidt & \\
\hline & The Bells Of Rhymney & 1965 & Idris Davies - Pete Seeger & \\
\hline & Rock Salt And Nails & 1965 & Bruce "U. Utah" Philips & Rosalie Sorrels \\
\hline \multirow{11}{*}{ Folk } & Bonnie Ship The Diamond & & & \\
\hline & $\begin{array}{l}\text { Come All Ye Far And Tender } \\
\text { Ladies }\end{array}$ & & & \\
\hline & Coming Round The Mountain & & & \\
\hline & Down On Me & & & \\
\hline & Going Down The Road & & & \\
\hline & Hills Of Mexico & & & \\
\hline & Johnny Todd & & & \\
\hline & 900 Miles & & & \\
\hline & Ol' Roisin The Beau & & & \\
\hline & Wildwood Flower & 1928 & A. P. Carter & Carter Family \\
\hline & Young but Daily Growing & & & \\
\hline
\end{tabular}

I@J vol.1, no.1 (2010) http://www.iaspmjournal.net 
Tenendo presente tale approccio teorico appare chiaro come il comportamento mantenuto da Dylan \& The Hawks nei Basement Tapes non sia basato solamente su processi ripetitivi, ma ha sicuramente a che fare con alterazioni e sviluppi del materiale originale. Ciò è necessario per rendere la nuova creazione un oggetto realmente diverso dalle fonti dalle quali trae ispirazione, dimostrando di lavorare in gran parte sulla dimensione storica, intensionale, della composizione. Tramite questa serie di relazioni intertestuali una canzone riesce a dare forma al proprio messaggio, riprendendo e sviluppando i propri referenti fino a farli diventare parte di un costrutto indipendente da essi. Secondo questa prospettiva 'Clothesline Saga' sarebbe un pezzo in diretta connessione con 'Ode to Billie Joe' non perché ne ripercorra fedelmente le caratteristiche strutturali, bensì perché è una canzone basata sugli stessi fondamenti organizzativi profondi, come evidenziato già dal sottotitolo: 'Answer to Ode'. Le due canzoni, infatti, presentano elementi fondamentalmente comuni, ma declinati in maniera leggermente diversa:

- in entrambi i casi la singola strofa è riconducibile a un'organizzazione di stampo blues $A A B$, con le prime due sezioni $A$ dedicate alla presentazione di una situazione statica e B a introdurre l'azione (v. Tabella 5). Inoltre, i due testi verbali possono anche essere accomunati per la condivisione dello stesso schema rimico AABBCC e di un'attitudine quasi "documentaria", testimoniata dalle date menzionate precisamente nei versi delle canzoni;

- $\quad$ il vocabolario armonico delle due canzoni è basato sugli accordi di tonica, sottodominante e dominante (v. Tabella 6). In 'Clothesline Saga' questo dà luogo a una semplice aumentazione da 12 a 24 battute del pattern blues standard, mentre 'Ode to Billie Joe' è basata su una tonicizzazione dell'accordo di dominante che ne modifica la struttura blues di conseguenza, pur giocando con gli stessi materiali di base;

Tabella 5: Le strofe iniziali di 'Clothesline Saga' e 'Ode to Billie Joe' interpretate secondo lo schema $A A B$.

\begin{tabular}{|l|l|}
\hline $\begin{array}{l}\text { After a while we took in the clothes / And nobody said very much } \\
\text { Just some old wild shirts and a couple pairs of pants / Which nobody really wanted to touch }\end{array}$ & A \\
\hline $\begin{array}{l}\text { Mama come in and picked up a book / An' Papa asked her what it was } \\
\text { Someone else asked, "What do you care?" / Papa said, "Well, just because" }\end{array}$ & A \\
\hline $\begin{array}{l}\text { Then they started to take back their clothes / Hang 'em on the line } \\
\text { It was January the thirtieth / And everybody was feelin' fine }\end{array}$ & B \\
\hline
\end{tabular}

It was the third of June, another sleepy, dusty Delta day.

I was out choppin' cotton and my brother was balin' hay.

And at dinner time we stopped, and we walked back to the house to eat.

And mama hollered at the back door "y'all remember to wipe your feet."

And then she said she got some news this mornin' from Choctaw Ridge

Today Billy Joe MacAllister jumped off the Tallahatchie Bridge. 
Tabella 6: Le concatenazioni di 'Clothesline Saga' e 'Ode to Billie Joe' a confronto.

\begin{tabular}{|l|l|l|l|}
\hline$\|: E$ & $E$ & $E$ & $E: \|$ \\
\hline A & A & A & A \\
\hline E & E & E & E \\
\hline B & B & B & B \\
\hline E & E & E & E \\
\hline
\end{tabular}

\begin{tabular}{|l|l|l|l|}
\hline$\|: D$ & D & D & D : \\
\hline G & G & G & G \\
\hline D & D & D & D \\
\hline G & G & G & G \\
\hline D & C & D & D \\
\hline
\end{tabular}

- $\quad$ la linea melodica è modellata in entrambe le canzoni seguendo un principio statico, con uso esclusivo di intervalli di seconda o di terza ( $\mathrm{v}$. Figura 2). E' anche importante da notare, sotto il profilo melodico, come 'Clothesline Saga' e 'Ode to Billie Joe' siano cantate con una voce dalla "grana" molto simile, caratterizzata da un'intonazione piatta e fredda, quasi a voler allontanare il cantante dalla partecipazione diretta alla storia che sta raccontando al suo pubblico.

D

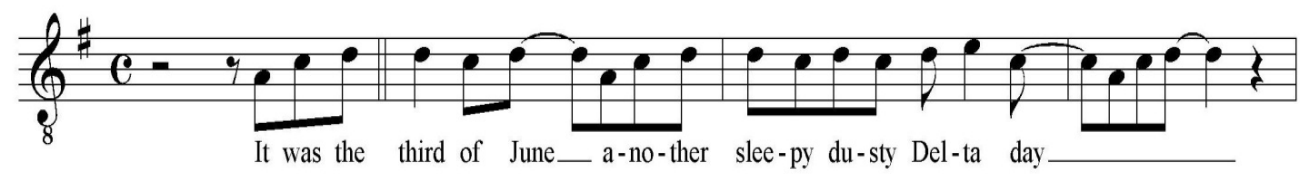

$E$

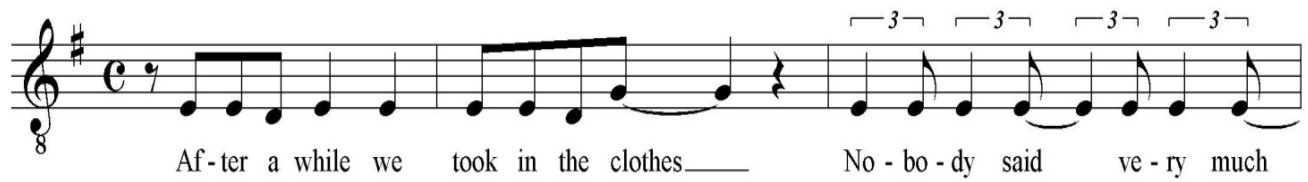

Figura 2: 'Ode to Billie Joe' e 'Clothesline Saga', prime battute della melodia vocale. 


\section{3. 'Lo and Behold' e I'utopia degli Stati Uniti secondo Bob Dylan}

II terzo esempio scelto per la mia indagine sui Basement Tapes è 'Lo and Behold', una canzone che ha avuto l'effetto principale di spostare il focus principale del discorso dalla dimensione puramente musicale al suo contenuto verbale. Ispirata al tema del viaggio, la narrazione si snoda attraverso un itinerario in treno tra diverse città degli Stati Uniti, sfruttando topos ricorrente nella cultura americana. Nella Figura 3 è possibile osservare il percorso descritto da Dylan: un'andata e ritorno da San Antonio a Pittsburgh, passando da Memphis. II protagonista, in altre parole, viaggia lungo l'intera Costa Est della nazione, da un estremo all'altro, dal confine col Messico alla regione dei Grandi Laghi. E' questa l'area dove si stabilirono la gran parte dei coloni provenienti dall'Europa, per poi spostarsi gradualmente verso ovest. Questa parte dell'America è il luogo dove si è formata l'autentica cultura americana, come prodotto delle varie fonti, europee ed africane, che hanno contribuito a modellarne le fattezze. Oltre a ciò, è indicativo dell'orizzonte cui Dylan si riferisce il fatto che l'itinerario sia percorso proprio in treno, un oggetto importantissimo nella popular culture americana fino ai giorni nostri, che cominciò ad acquisire i propri connotati simbolici alla fine del XIX secolo. Durante questo periodo il mito della ferrovia si legò indissolubilmente a quello di una nazione in costante espansione mentre, in parallelo, la rapida espansione delle principali città può essere interpretata alla luce della più semplice connessione tra luoghi lontani, con una serie di ricadute sotto il profilo culturale e musicale facilmente immaginabili. In un tale contesto, non sarà allora una sorpresa constatare che tutti i luoghi nominati dal cantante in 'Lo and Behold' siano inclusi nell'area degli Stati Uniti nella quale il sistema ferroviario si sviluppò prima, favorendo processi di ibridazione e scambio più profondi qui rispetto al resto del paese. ${ }^{5}$

Capire come è trattato l'argomento dell'identità americana in questa canzone può portare a delineare con maggiore chiarezza in che modo questo tema sia concepito da Dylan, come lui interpreti l'idea di "americanità". E' questo un punto cruciale in un insieme di registrazioni definite da Greil Marcus (2002) una "mappa", dalla quale emerge un nuovo tipo di canzone americana e un'utopia nazionale inedita, esattamente nella fase della carriera del cantante segnata dall'allontanamento dalle speranze in un prossimo, radicale cambiamento sociale. Ciò è segnalato dall'estremo idealismo della sua posizione politica, che lascia consapevolmente da parte istanze realistiche, come se si volesse solamente tratteggiare un percorso teorico per realizzare un'entità sociale impossibile in pratica. ${ }^{6}$ Guardando alla confusione diffusa nella società e nella politica americana alla metà degli anni Sessanta, quello che Dylan sembra cercare in una visione simile è il concetto di una nazione risultante dalla fusione di esperienze, coscienze e aspirazioni dissimili, mescolate insieme attraverso l'appartenenza a un unico corpo nazionale. Nella democrazia ideale tratteggiata in queste canzoni, insomma, gli eroi delle ballads folk e i freaks al centro di canzoni come 'Apple Suckling Tree' o 'Lo and Behold' possono vivere fianco a fianco. Riemerge da questo punto di vista un germe con ogni probabilità risalente 
al periodo folk-revival di Dylan: un'ideale comunità che riesca a includere tutti i suoi membri all'interno di un unico movimento culturale di stampo generalmente democratico, progressista.

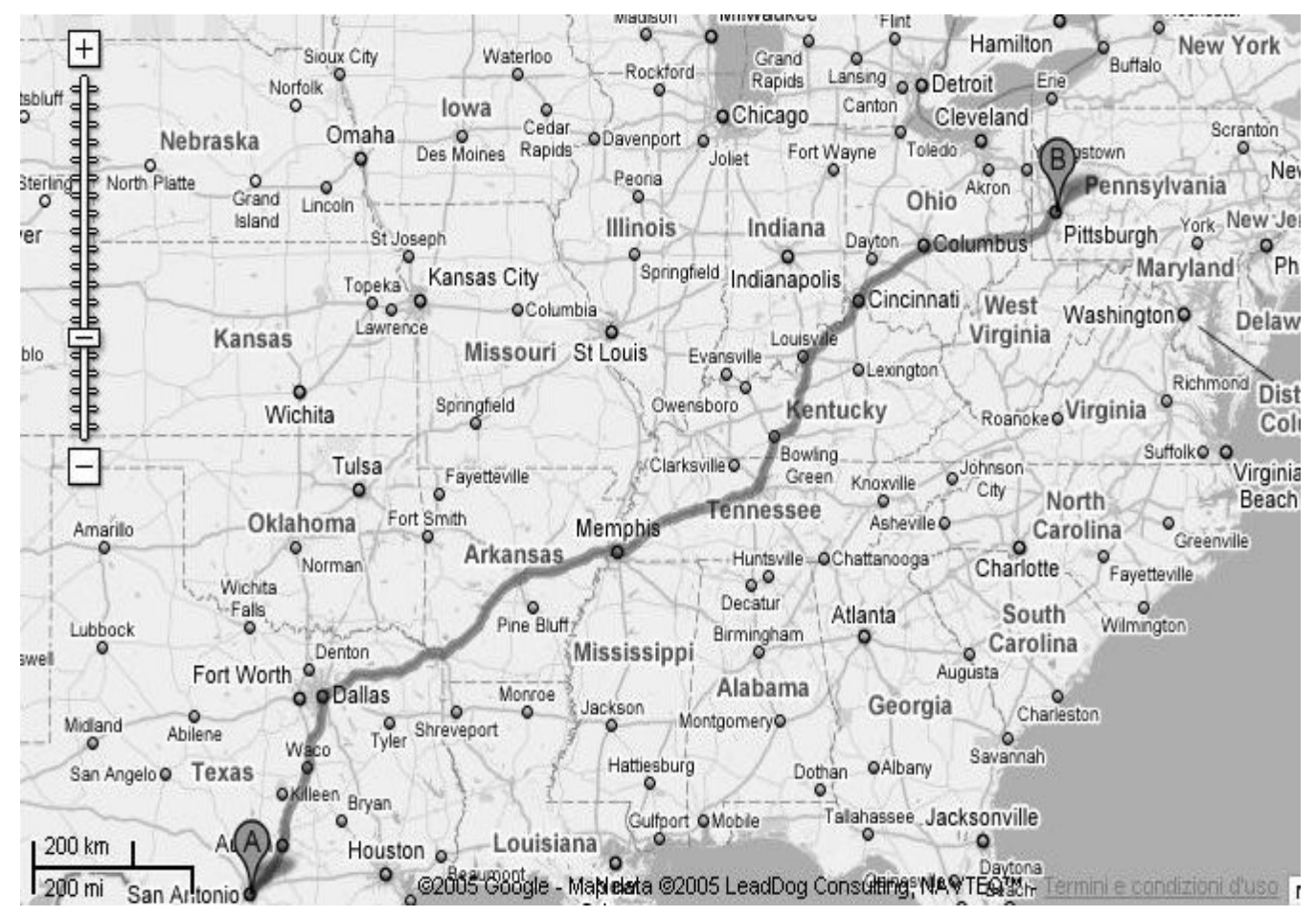

Figura 3: L'itinerario di 'Lo and Behold' lungo la Costa Est degli Stati Uniti.

Oltre a considerazioni di carattere ideologico, questa canzone dà anche l'opportunità di mostrare un esempio significativo delle potenzialità di un'analisi che prenda in considerazione, per quanto con strumenti concettuali molto semplici e intuitivi, anche l'aspetto puramente sonoro delle due versioni di 'Lo and Behold' testimoniate dal bootleg The Genuine Basement Tapes (1990). Nonostante le due registrazioni, dal punto di vista organizzativo, siano molto simili, il loro profilo generale quando sono aperte con un audio editor digitale è differente, come mostrato nella Figura 4. La prima immagine riproduce il percorso di una canzone con un livello di decibel costante per la sua intera durata, mentre nella seconda si può osservare all'opera un climax sonoro comprendente l'intero brano. In questo caso i musicisti stanno lavorando su un tipo di struttura a largo raggio non riscontrabile tramite un esame dei parametri rappresentabili in notazione musicale, ma solamente concentrando l'attenzione sulla dimensione propriamente aurale del pezzo registrato, della composizione intesa come traduzione su nastro magnetico di una data performance. 


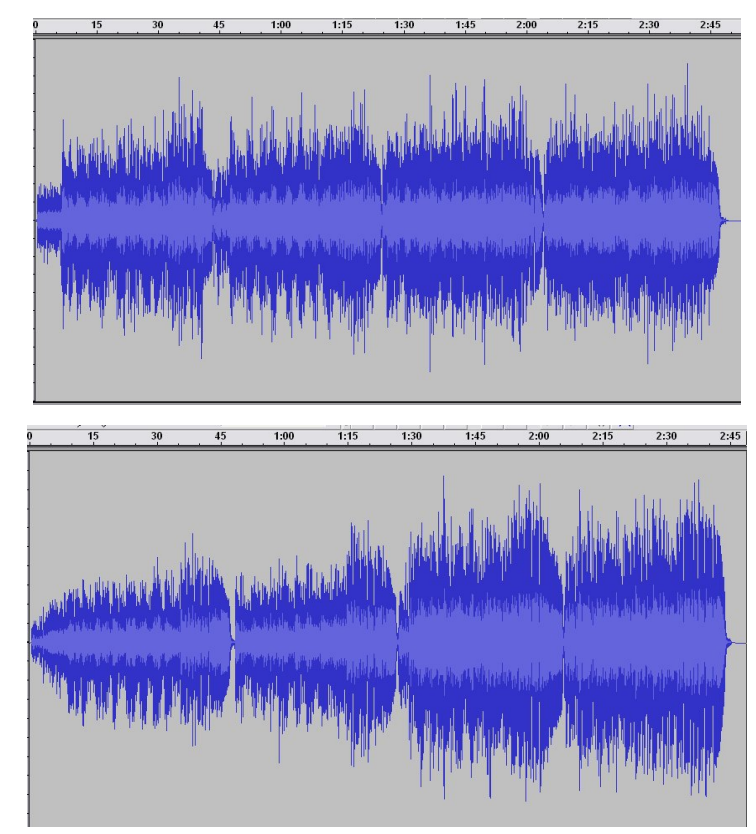

Figura 4: Rappresentazione grafiche di 'Lo and Behold' \#1 e \#2 (realizzate con il software Audacity).

\section{4. 'Tears of Rage' e le dinamiche compositive nelle canzoni popular}

L'ultimo esempio analizzato, 'Tears of Rage', mi ha dato modo di confrontarmi con uno dei pochi pezzi presenti in tre diverse interpretazioni nel bootleg utilizzato quale fonte principale per il mio studio, permettendomi di esaminare nel dettaglio la graduale elaborazione di un particolare numero del repertorio. Mi sono potuto così avvicinare a un momento normalmente difficile da esaminare nella popular music, cioè quello della composizione, a causa della limitata disponibilità di fonti dirette antecedenti il risultato pubblicato su disco. Nel caso di 'Tears of Rage' l'occasione di poter lavorare su tre versioni della stessa canzone ha rivelato la stretta coerenza complessiva del progetto compositivo dietro al pezzo. Dai cambiamenti e dalle varianti presenti nelle varie incisioni (anche di altri esempi tratti dallo stesso repertorio) si conferma la volontà dei musicisti di concentrarsi soprattutto sulla canzone come forma espressiva, e di modificarne gli elementi macro- e micro-strutturali soprattutto in vista delle sue necessità comunicative, senza privilegiare necessariamente uno o l'altro livello.

Per favorire un confronto tra le diverse incisioni di 'Tears of Rage', la Tabella 7 riassume le loro principali coordinate strutturali, raccogliendo in un'unica soluzione la rappresentazione della dimensione formale (nelle colonne) e della scatola sonora (evidenziate dalle linee di diversa consistenza sui canali destro e sinistro). Dalla comparazione tra queste tre versioni è possibile avanzare alcune osservazioni utili per definire il processo attraverso cui la canzone prende forma: 
- l'introduzione viene ridotta progressivamente da quattro a una battuta, precisando la stessa idea musicale in un lasso di tempo più ristretto;

- la durata della strofa è la stessa in tutte le versioni, ma il ritornello solo nell'ultima versione arriva a un'estensione costante: nella prima versione era una sezione da otto, nove o dieci battute, da otto o nove nella seconda, e solo nella terza si conforma a un impianto regolare di otto battute;

- $\quad$ in 'Tears of Rage' \#2 si possono trovare elementi scartati dalla prima e dalla terza versione, a iniziare dal tempo ternario e dalla presenza del coro solamente nel ritornello. Nelle versioni \#1 e \#3, invece, il tempo è binario e la parte vocale di controcanto, affidata a Richard Manuel e Rick Danko, inizia i suoi vocalizzi circa a metà della strofa, intensificando gradualmente il volume sonoro e addolcendo il passaggio tra le due principali sezioni macroformali;

- in 'Tears of Rage' \#3, quella definitiva che si ritroverà nella pubblicazione ufficiale del 1975 , il coro ha un volume più alto rispetto alle altre incisioni, confermando il suo ruolo strutturale all'interno della composizione nel suo complesso.

Le dinamiche implicate dalle trasformazioni di 'Tears of Rage' non rappresentano un fenomeno isolato, ma riflettono una situazione diffusa all'interno del repertorio considerato. Dopo aver compiuto una ricognizione dettagliata sull'intero patrimonio di canzoni originali elaborate a Woodstock nel 1967, è emerso chiaramente come anche la restante parte delle incisioni sia stata sottoposta a un graduale processo di selezione e modifica. Per arrivare a questo risultato analitico, è stato fondamentale confrontarsi con una testimonianza, come quella del bootleg The Genuine Basement Tapes, precedente alle operazioni di missaggio e masterizzazione necessarie per mettere il disco sul mercato nella sua edizione ufficiale del 1975. Un'analisi fondata su questo tipo di materiale ha, infatti, permesso di identificare con maggiore chiarezza quali criteri siano alla base di determinate scelte compositive, e come i diversi livelli strutturali siano reciprocamente collegati. Tutte queste considerazioni fanno trasparire la presenza di una precisa progettualità alla luce della quale poter interpretare globalmente le diverse incisioni di una canzone, come approssimazioni successive rispetto a un modello ideale. Tale costrutto, concepito a priori dal suo o dai suoi autori, va a costituire la sostanza profonda della composizione, costruendo un sistema attraverso il quale sono collegate le caratteristiche più diverse. Da questo punto di vista, mi auguro che le osservazioni condotte sul caso particolare dei Basement Tapes possano offrire una serie di coordinate teoriche utili per un'applicazione a repertori popular anche distanti a livello cronologico o geografico, ponendosi come limitato contributo a una più ampia comprensione delle dinamiche compositive di musiche basate su processi di natura non scrittoria. 
Tabella 7: 'Tears of Rage' \#1/3, organizzazione strutturale.

\begin{tabular}{|c|c|c|c|c|c|c|c|c|c|c|c|c|c|c|}
\hline & Battute & $1 / 4(4)$ & & (16) & $21 / 3$ & (10) & & $1 / 46(1$ & & $47 / 55(9)$ & & $6 / 71(16$ & & $72 / 29(8)$ \\
\hline & & & $5 / 8$ & $9 / 20$ & $21 / 28$ & $29 / 30$ & $31 / 33$ & $34 / 38$ & $39 / 46$ & & $56 / 57$ & $58 / 64$ & $65 / 71$ & \\
\hline & Armonia & & & $A$ & & & & A & & B & & A & & B \\
\hline & Piano & & & & & & & & & & & & & \\
\hline$L$ & Chitarra 2 & & & & & & & & & & & & & \\
\hline & Basso & & & & & & & & & & & & & \\
\hline & Cori & & & & --- & & & & --- & ---- & & & -- & $----\cdot$ \\
\hline $\boldsymbol{R}$ & Chitarra 1 & --- & & -- & & -- & & --- & & ----- & & - & & ---- \\
\hline
\end{tabular}

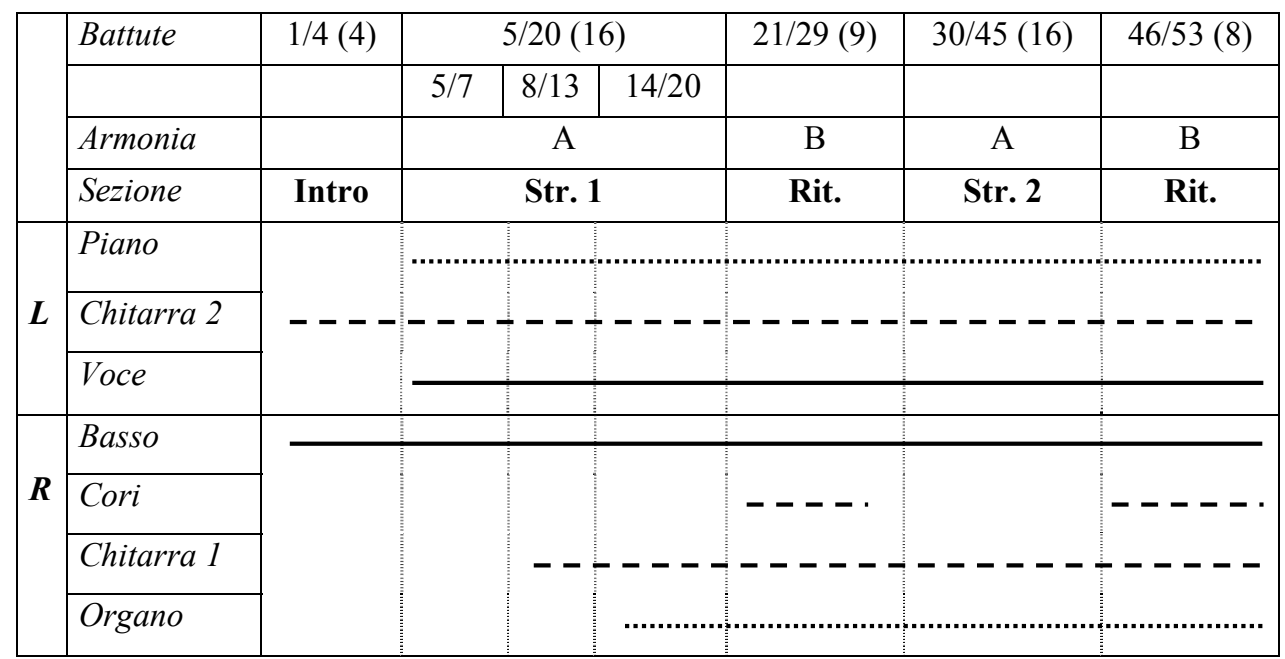

\begin{tabular}{|c|c|c|c|c|c|c|c|c|c|c|c|}
\hline & Battute & $1(1)$ & $2 / 17(16)$ & $18 / 25(8)$ & $26 / 41$ & (16) & $42 / 49(8)$ & $50 / 6$ & (16) & $66 / 73(8)$ & $74(1)$ \\
\hline & & & & & $26 / 31$ & $32 / 41$ & & $50 / 57$ & $58 / 65$ & & \\
\hline & Armonia & & A & B & $\mathrm{A}$ & & B & & & $\mathrm{B}$ & \\
\hline & Sezione & Intro & Str. 1 & Rit. & Str. & & Rit. & St & & Rit. & Coda \\
\hline$I$ & Piano & & & & & & & & & ................. & \\
\hline & Chitarra 2 & & & & & & & & & & \\
\hline & Voce & & & & & & & & & & \\
\hline & Basso & & & & & & & & & & \\
\hline$R$ & Cori & & & & & & & & & & \\
\hline & Chitarra 1 & & & & & & & & & & \\
\hline & Organo & & & & & & & & & & \\
\hline
\end{tabular}




\section{Notes}

1. Una simile presa di posizione estetica è un esempio di quella "negoziazione delle relazioni tra passato e presente" discussa da Zak (2004) confrontando le versioni di Dylan e di Hendrix di All Along the Watchtower.

2. II concetto di "scatola sonora", come rappresentazione tridimensionale delle caratteristiche sonore complessive di un brano, è tratta da Moore (2001, pp. 120129).

3. 'Froggie Went A-Courtin" sarà poi registrata dallo stesso Dylan più di 25 anni più tardi, nell'album World Gone Wrong (1993).

4. In accordo con quanto scritto da Kubik (1999, p. 125), nella musica americana derivata da fonti africane, un "area flessibile di altezze" è un caratteristica peculiare di sistemi scalari nei quali "valori di altezza differenti sono percepiti dal cantante come varianti di un unico tonema". Questo è molto comune proprio nel blues, genere nel quale entrambe le specie di terza sono spesso compresenti nello stesso pezzo. Considerando la notevole influenza di tale linguaggio nei
Basement Tapes, non sorprende di ritrovare anche in queste canzoni peculiarità simili, persino in questo esempio di canzone ibrida, a metà tra la tradizione blues (in special modo per le caratteristiche formali) e folk.

5. Come ha riconosciuto Katherine Preston (1998, p. 218) nel suo resoconto sullo sviluppo della musica americana del XIX secolo, il sistema ferroviario giocò un ruolo fondamentale nel disseminare le più diverse tendenze culturali fin nelle regioni più remote degli Stati Uniti. Una panoramica sull'immagine del treno e dei suoi significati nella musica popular e d'arte è offerta da un recente volume di Paolo Prato (2003), in larga parte dedicato ai repertori americani non di ambito colto.

6. A proposito della ritrosia di Dylan ad occuparsi di politica in questi anni, Mike Marqusee (2993, p. 268) l'ha definita un fallimento "of the Vietnam test posed to all Americans. He did turn away his head and pretend that he just didn't see [...]".

\section{References}

Bowman, Rob. 2002. "Il sound Stax: un'analisi musicologica", Rivista di Analisi e Teoria Musicale, Vol. 8, No. 2, pp. 3-44.

Chester, Andrew. 1970. "For a Rock Aesthetic", New Left Review, No. 59, pp. 8396.

Covach, John. 2001. "Popular Music, Unpopular Musicology" in Cook, N. \& Everist, M. eds. Rethinking Music, Oxford University Press, Oxford, pp. 452-470.

Kubik, Gerhard. 1999. Africa and the Blues, University Press of Mississippi, Jackson.

La Via, Stefano. 2006. Poesia per musica e musica per poesia. Dai trovatori a Paolo Conte, Carocci, Roma.

Marcus Greil. 1997. Invisible Republic. Bob Dylan's Basement Tapes, Henry Holt \& C, New York. 
Marqusee, Mike. 2003. Wicked Messenger. Bob Dylan and the 1960s, Seven Stories, New York.

Merwe, Peter (Van Der). 1994. Origins of the Popular Style: The Antecedents of Twentieth-Century Popular Music, Clarendon Press, Oxford.

Middleton, Richard. 1994. Studiare la popular music, Feltrinelli, Milano.

Middleton, Richard. 2002. "Analisi della popular music e musicologia: gettare un ponte?", Rivista di Analisi e Teoria Musicale, Vol. 8, No. 2, pp. 115-131.

Moore, Allan. 1997. "Anachronism, Responsibility and Historical Intension", Critical Musicology,

<http://www.leeds.ac.uk/music/Info/critmus/articles/1997/03/01.html> [Accessed 13 May 2010]

Moore, Allan. 2001. Rock: The Primary Text. Developing a Musicology Of Rock, Ashgate, Aldeshot. Second edition.

Oxford Music Online. 2009. "Blues" in The New Grove Dictionary Of Music And Musicians. 2nd Edition, 20 April. <http://www.oxfordmusiconline.com/public > [Accessed 13 May 2010]

Prato, Paolo. 2003. II treno dei desideri. Musica e ferrovia da Berlioz al rock, L'Epos, Palermo.

Preston, Katherine. 1998. "Art Music from 1800 to 1860" in David Nicholls ed. The Cambridge History of American Music, Cambridge University Press, Cambridge, pp. 186-213.

Tagg, Philip. 1994. "Analizzare la popular music: teoria, metodo e pratica" in Roberto Agostini - Luca Marconi eds. Popular music. Da Kojak al rave, Clueb, Bologna, 1994, pp. 43-75.

Zak, Albin J. III. 2004. "Bob Dylan and Jimi Hendrix: Juxtaposition and Transformation in All Along the Watchtower", Journal of American Musicological Society, Vol. 57, No. 3, pp. 599-644.

\section{Discography}

Beatles, The. 1967. Sgt. Pepper's Lonely Hearts Club Band, Parlophone, 1 giugno, UK.

Dylan, Bob. 1967. John Wesley Harding, Columbia, 27 dicembre, USA.

Dylan, Bob. 1969. Nashville Skyline, Columbia, 9 aprile, USA.

Dylan, Bob. 1993. World Gone Wrong, Columbia, 26 ottobre, USA.

Dylan, Bob (with The Band). 1975. The Basement Tapes, Columbia, 25 giugno, USA.

Dylan, Bob (with The Band). 1990. The Genuine Basement Tapes, Scorpio, USA. Gentry, Bobbie. 1967. Ode to Billie Joe, giugno, Capitol, USA.

Hendrix, Jimi. 1968. Electric Ladyland, Reprise, 16 settembre, USA. 